\title{
Priorities in astronomy
}

US astronomers are being forced to revisit their widely admired system for ranking large projects.

$\mathrm{n}$ the beginning, astronomers believe, our tiny Universe underwent 'inflation' - a period of growth so rapid that it separated sections of the cosmos from each other forever.

Something similar has happened in astronomy itself over the past half-century. In the 1950s, the field was a primordial ball of scientists using a handful of modest telescopes to peer at stars and galaxies. Today, thousands of them use telescopes, radio arrays, balloons and satellites to learn about black holes, extrasolar planets, star nurseries or the early Universe.

In the United States, a process known as the decadal review has guided this rapid expansion. The review is compiled by a committee, gathered under the auspices of the National Academy of Sciences, and lists which instruments should be built over the next decade, along with estimates of their cost. It has served US astronomy well, and other capital-intensive disciplines of science have sought to learn from its example. But the review process is under increasing strain (see page 386). Most projects in the 2001 review have been severely delayed, and some have been "indefinitely deferred". As the next decadal review gets under way, astronomers are wondering how to salvage its tattered credibility.

The problem arises, first and foremost, because astronomy funding's own period of inflation is drawing to a close. Budget constraints at federal science agencies, most notably NASA, have seen to that. The slowdown is compounded by the steadily growing size, and therefore cost, of the decadal review's project wish-list.

And as happened in the early Universe, the rapid early expansion of astronomy has left its component parts cut off from one another. Astronomers have organized themselves by the type of starlight they study - X-rays are one subdiscipline, infrared another. The partition made sense because each subfield requires different types of telescopes. But it has added a sectarian element to the review process, as various subpanels, organized by light type, have fought to secure the place of their field's instrument in the review document.

The need to placate these different groups has forced the review's outcome to become unwieldy. In 1972, for example, the review simply listed eleven favoured projects in rank order. By 2001, it was placing projects in subcategories of large, medium and small, and whether they were space- or ground-based, before ranking them. This obtuse system identified no less than five different projects as astronomy's 'top priority'.

Astronomers are therefore looking at ways to restore some of the simplicity that was the review's great strength. One approach may be to turn to the science. Rather than dividing astronomy by wavelength, reviewers could decide which areas of investigation are likely to prove most promising - the study of 'dark energy', for example. Researchers from each subfield could then come together on subpanels to establish which instruments could best address the problem, and how each subfield could best contribute.

The basic idea is not without prece-

"Astronomers are
looking for ways
to restore some of
the simplicity that
was the review's
great strength."

dent: US Earth scientists are already using it in their own, inaugural decadal review. Their National Academies panel is organized by categories such as weather, climate, hydrology, natural hazards and solid Earth research, with the weather panel, for example, incorporating hydrologists, atmospheric physicists and oceanographers. It expects to produce a prioritized list of instruments, some of which will be shared by subdisciplines.

Such a change in outlook is not a panacea for astronomy. Given the budget constraints, the next review will have to make tough choices and exclude some excellent projects. But reorganizing the decadal review along scientific lines would go some way towards ensuring its continued relevance in astronomy's post-inflationary epoch.

\section{A German academy}

In seeking to build a credible national academy from scratch, Germany faces a tough challenge.

ए or historical reasons, modern Germany has no national scientific academy along the lines of the Royal Society in London or the US National Academies. It is now seeking to design one - but building a reputable academy from scratch may be more difficult than it at first appears.

Clearly, the academy's value will ultimately be measured by the quality of the reports and recommendations it produces, and by the influence it manages to yield. In a pluralistic society, no single organization can claim to be a central committee of truth. But the functioning of modern nations correlates closely with their handling of technical, medical and scientific problems. They need to receive well-considered scientific advice that has a real chance of making a difference in the political arena.

The fact that Germany is building an academy from scratch gives it the opportunity to learn from others, and to do it right. Scientific academies have sometimes carried the whiff of exclusive clubs where scientists who are usually elderly, white and male retire to indulge in arcane intellectual pleasures. It is a tough balancing act to ensure that an academy will respect experience and acquired wisdom, while also reflecting the diversity - in age, gender, background and outlook - of today's scientific community.

Germany's existing learned societies include seven regional academies, which are most active in the humanities and social sciences, and the 350-year-old Leopoldina, which represents the medical and 